\title{
Overall Tracer Uptake Compared to Baseline
}

National Cancer Institute

\section{Source}

National Cancer Institute. Overall Tracer Uptake Compared to Baseline. NCI Thesaurus.

Code C139128.

A visually assessed combination of extent and intensity of tracer uptake, as compared to baseline. 